\title{
Layer-by-Layer Assembly of Chitosan and Recombinant Biopolymers into Biomimetic Coatings with Multiple Stimuli-Responsive Properties
}

\author{
Rui R. Costa, * Catarina A. Custódio, Francisco J. Arias, José C. Rodríguez-Cabello, \\ and João F. Mano*
}

In this work, biomimetic smart thin coatings using chitosan and a recombinant elastin-like recombinamer (ELR) containing the cell attachment sequence arginineglycine-(aspartic acid) (RGD) are fabricated through a layer-by-layer approach. The synthetic polymer is characterized for its molecular mass and composition using mass spectroscopy and peptide sequencing. The adsorption of each polymeric layer is followed in situ at room temperature and pH 5.5 using a quartz-crystal microbalance with dissipation monitoring, showing that both polymers can be successfully combined to conceive nanostructured, multilayered coatings. The smart properties of the coatings are tested for their wettability by contact angle (CA) measurements as a function of external stimuli, namely temperature, $\mathrm{pH}$, and ionic strength. Wettability transitions are observed from a moderate hydrophobic surface (CAs approximately from $62^{\circ}$ to $71^{\circ}$ ) to an extremely wettable one (CA considered as $0^{\circ}$ ) as the temperature, $p H$, and ionic strength are raised above $50^{\circ} \mathrm{C}, 11$, and $1.25 \mathrm{M}$, respectively. Atomic force microscopy is performed at $\mathrm{pH} 7.4$ and $\mathrm{pH} 11$ to assess the coating topography. In the latter, the results reveal the formation of large and compact structures upon the aggregation of ELRs at the surface, which increase water affinity. Cell adhesion tests are conducted using a SaOs-2 cell line. Enhanced cell adhesion is observed in the coatings, as compared to a coating with a chitosan-ending film and a scrambled arginine-(aspartic acid)-glycine (RDG) biopolymer. The results suggest that such films could be used in the future as smart biomimetic coatings of biomaterials for different biomedical applications, including those in tissue engineering or in controlled delivery systems.

R. R. Costa, C. A. Custódio, Dr. J. F. Mano

3B's Research Group-Biomaterials, Biodegradables and Biomimetics University of Minho

AvePark, Zona Industrial da Gandra

São Cláudio do Barco

4806-909 Caldas das Taipas, Guimarães, Portugal

E-mail: rui.costa@dep.uminho.pt; jmano@dep.uminho.pt

R. R. Costa, C. A. Custódio, Dr. J. F. Mano

ICVS/3B's - PT Government Associate Laboratory

Braga/Guimarães, Portugal

DOI: $10.1002 /$ smll.201100875
Dr. F. J. Arias, Dr. J. C. Rodríguez-Cabello

G.I.R. Bioforge

University of Valladolid

Edifício I+D, Paseo de Belén, 1, 47011, Valladolid, Spain

Dr. F. J. Arias, Dr. J. C. Rodríguez-Cabello

Networking Research Center on Bioengineering

Biomaterials and Nanomedicine (CIBER-BBN), Valladolid, Spain 


\section{Introduction}

The modification of surfaces has been a key aspect in biology and biotechnology, including cell expansion, biomaterials development, and preparation of substrates for regenerative medicine. ${ }^{[1]}$ In the field of implantable devices, the surface is the first contact with the organism and dictates subsequent biological events, namely the cellular behavior and biointegration. Thus, surface engineering is of utmost significance for the conception of devices with an improved biological performance..$^{[2]}$

Surface modification has also been implemented to produce stimuli-responsive surfaces for a variety of applications, including those in biomineralization control, ${ }^{[3]}$ cell-sheet engineering, ${ }^{[4]}$ surfaces with extreme wettability transitions, ${ }^{[5]}$ and microfluidics ${ }^{[6]}$ mostly based on variations of the hydration state of deposited polymeric films. Usually, the modification is performed by grafting stimuli-responsive polymers onto a substrate, which are typically synthetic and non-biodegradable, such as poly $\left(N\right.$-isopropylacrylamide) (PNIPAAm) ${ }^{[7]} \mathrm{A}$ good example of the latter is the work developed by Okano et al. regarding cell-sheet engineering. ${ }^{[4 b]}$

In more recent years, emphasis has been given to nonharmful and versatile techniques to modify polymeric substrates: the sequential adsorption of proteins and polysaccharides, known as layer-by-layer (LbL) adsorption, is one of the most promising today. The principle behind LbL adsorption lies in the existence of multiple intermolecular interactions, such as electrostatic contacts, hydrophobic interactions, and hydrogen bonding, where the cooperative effects of multipoint attractions play the most important role. It is a simple and versatile technique that allows the production of robust coatings, even in substrates with complex geometries; because it discards the need for organic and harmful solvents, it is an attractive technique for tissue engineering applications. ${ }^{[8]}$ Multilayer systems have already been proposed for different biomedical applications, including biomimetic composite-like coatings, the control drug release, or the manipulation of adhesion, differentiation, proliferation and even function of attached cells. ${ }^{[9]}$

In this work, we plan to conceive coatings using natural and nature-inspired macromolecules in a multilayered fashion using the LbL approach at mild processing conditions. Natural materials are intricate and multifunctional structures that often inspire scientists to design novel classes of materials. ${ }^{[10]}$ It is the case of elastin-like recombinamers (ELRs), also designated elastin-like polymers (ELPs). This family of peptide-based polymers was developed by Urry, relying in genetic engineering techniques, and they have their basic structure rooted in elastin, the extracellular elastic protein of higher animals. ${ }^{[11]}$ In aqueous environment they are known to have self-assembly behavior and exhibit a transition temperature $\left(T_{\mathrm{t}}\right)$ : below $T_{\mathrm{t}}$, the free polymer chains adopt random coil conformations; above it, they fold into an organized structure known as a $\beta$-spiral. Although ELRs respond primarily to temperature, the effects of other stimuli, such as $\mathrm{pH}$, ionic strength, and concentration, also affect the transition phenomenon. ${ }^{[12]}$ Their recombinant nature is one of their attractive properties because it allows tuning their physicochemical properties and introducing sequences to extend their functionality, including biologically relevant domains. ${ }^{[13]}$ The work of Girotti et al., who were able to biosynthesize an extracellular matrix analogue enclosing the cell attachment sequence arginine-(glutamic acid)-(aspartic acid)-valine (REDV), is an example of such versatility. ${ }^{[14]}$ Thus, ELRs are an attractive option over the most commonly used formulations in LbL adsorption.

Swierczewska et al. have previously reported the use of ELRs for LbL thin films. However, the peptides were modified and conjugated with either polyethyleneimine (PEI) or polyacrylic acid (PAA) to provide positive or negative charge, respectively. ${ }^{[15]}$ Our groups have also shown that ELRs can be successfully combined with polysaccharides to conceive coatings and some may even be applied using a multilayer approach. ${ }^{[16]}$ In order to explore the potential of ELR properties and the advantages of LbL, we intend to conceive nanostructured thin coatings made from the sequential adsorption of a polysaccharide and an ELR modified with the arginine-glycine-(aspartic acid) (RGD) cell adhesion motif, labeled ELR-RGD. RGD is expected to increase the cell adhesion in vitro and prove useful in the design of devices with tunable cell attachment or even improved host integration. We selected chitosan (CHI) because, aside from being a well-known biocompatible material, it is one of the few polysaccharides exhibiting positive charge (at acidic conditions), it is abundant, and its production is environmentally safe. ${ }^{[17]}$

The proposed system will also allow for expanding the concept of multilayered films to a new attractive class of peptide-based polymers, making them more robust than simple monolayers, and could bring new possibilities in the development of nanostructured coatings of materials in tissue engineering. The buildup of the multilayers will be monitored in real time using a quartz-crystal microbalance with dissipation monitoring (QCM-D). Distinct techniques, such as contact angle measurements, atomic force microscopy (AFM), and cell adhesion tests will be used to characterize it. We hypothesized that coatings containing this ELR may exhibit changes both in topography and wettability, depending on several stimuli, namely temperature, $\mathrm{pH}$, and ionic strength, and improved biological in vitro performance.

\section{Results and Discussion}

\subsection{ELR-RGD Characterization}

For the past two decades, much work has been done on the widely investigated poly(styrene sulfonate)/poly(allylamine hydrochloride) (PSS/PAH) system-considered so far as a model of multilayered thin films-and $\mathrm{CHI} /$ hyaluronan. ${ }^{[9 a}$ However, the buildup mechanism is not always straightforward for every material and distinct forces may influence the construction. ${ }^{[18]}$ Furthermore, coatings for tissue engineering applications should ideally contain other materials with more relevant features, including biological activity. It is the case of the biopolymer used in this work, which contains the RGD cell adhesion motif (Figure 1a). The ELR-RGD, 
a)

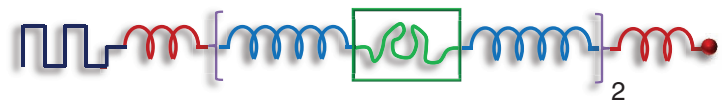

MGKKKP (VPGVG) ${ }_{14}\left\{\left(\right.\right.$ VPGIG) ${ }_{10}\left(\text { AVTGRGDSPASS) }(V P G I G)_{10}\right\}_{2}(V P G V G)_{14} V$

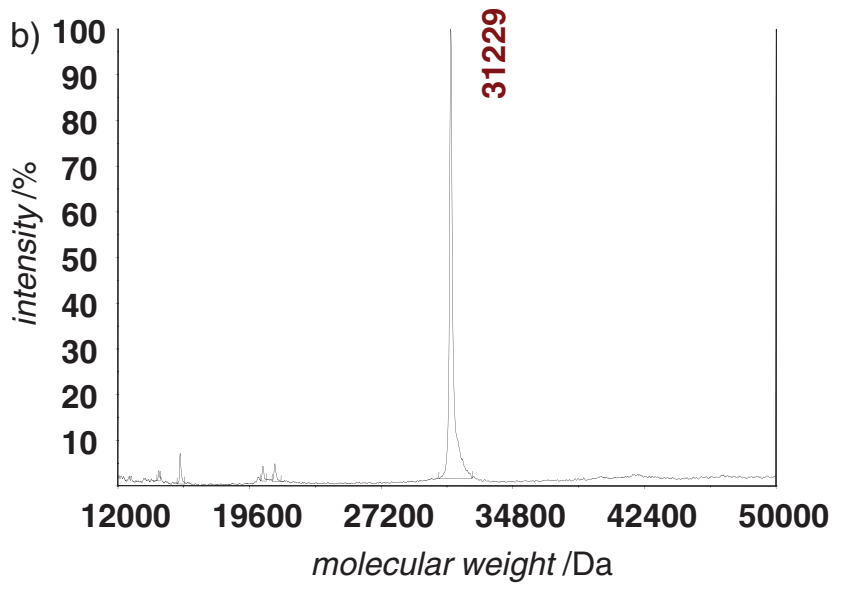

Figure 1. Characterization of the ELR-RGD. a) Schematic representation of the featured ELR chemical structure; b) MALDI-TOF spectrum of the biopolymer, with major peak at 31229 Da.

with 371 amino acids and a theoretical molecular weight of $31371 \mathrm{Da}$, contains 2 monomers of RGD, 2 aspartic acids, and 3 lysines isolated at the amino-terminal and exhibits a $T_{\mathrm{t}}$ of around $23^{\circ} \mathrm{C}$ in solution at physiological $\mathrm{pH}$ (data not shown). In contrast to our previous work, this ELR-RGD was engineered to allow electrostatic interactions during the multilayer buildup. ${ }^{[16 a]}$ In Figure 1b, a matrix-assisted laser desorption/ionization time-of-flight (MALDI-TOF) spectrum shows a high-intensity peak at $31229 \mathrm{Da}$, which is approximate to the theoretical value for this biopolymer.

In order to confirm if the produced ELR matched the theoretical amino acid composition, peptide sequencing tests were performed. In Table 1, it can be confirmed that the produced biopolymer composition matches well with the predicted amino acid composition. These results point to the bioproduction of a stock of ELR-RGD retaining the desired amino acid sequence.

\subsection{Analysis of CHI/ELR-RGD Film Buildup}

The ability of CHI and ELR-RGD to adsorb in a sequential fashion was first monitored by QCM-D. This technique is able to detect mass changes in the order of nanograms per square centimeter and measure the viscoelastic properties of the resulting surface. ${ }^{[19]}$ Due to the recombinant nature of ELRs, it is possible to tune their structure to appropriately fit a multilayer approach and design coatings for tissue engineering substrates.

Figure 2a shows the normalized frequency $\left(\Delta F_{\mathrm{v}} / v\right.$, where $v$ is the overtone) and dissipation $(\Delta D)$ variations for the fifth overtone $(25 \mathrm{MHz})$ after the construction of 20 layers onto gold-coated sensors. The $\Delta F_{v} / v$ decreased upon flushing the substrate with the sequence of polyelectrolytes, due to the deposition of polymer on the surface of the crystal, to
Table 1. Amino acid composition of the bioproduced ELR-RGD. ND = not determined.

\begin{tabular}{lcc}
\hline Amino acid & Predicted & Experimental \\
\hline Asp (D) & 2 & 2.27 \\
Thr (T) & 2 & 1.81 \\
Ser (S) & 6 & 4.7 \\
Pro (P) & 71 & 70.7 \\
Gly (G) & 141 & 141.48 \\
Ala (A) & 4 & 4.31 \\
Val (V) & 99 & 98.65 \\
Ile (I) & 40 & 39.6 \\
Lys (K) & 3 & 3 \\
Arg (R) & 2 & 2.18 \\
Met (M) & 1 & ND \\
\hline
\end{tabular}
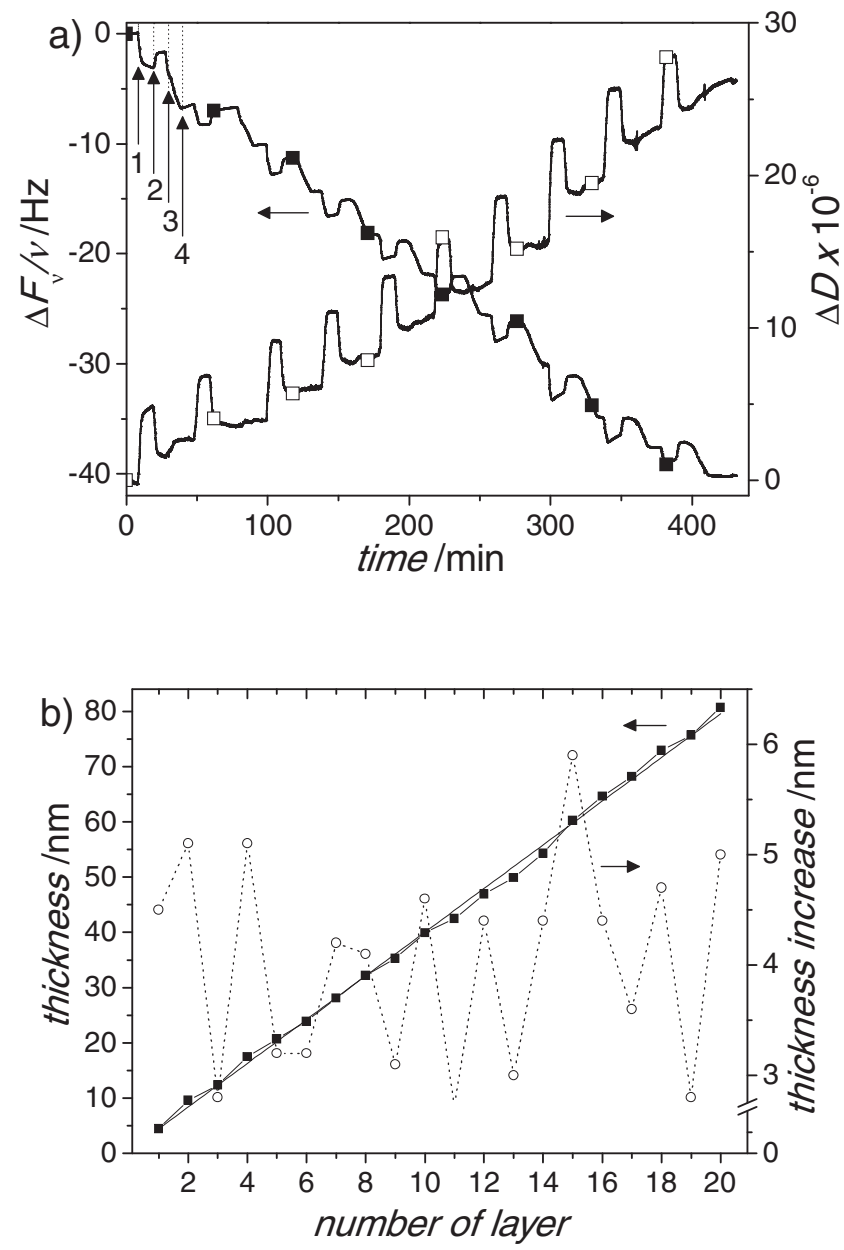

Figure 2. Buildup assessment of $\mathrm{CHI} / E L R-R G D$ films. a) QCM-D monitoring of normalized frequency $\left(\Delta F_{v} / v, \square\right)$ and dissipation $(\Delta D, \square)$ obtained at the fifth overtone. Numbers refer to adsorption of CHI (1), adsorption of ELR-RGD (3), and rinsing (2 and 4). b) Cumulative thickness evolution and thickness increase for the CHI/ELR-RGD film, for 20 layers, estimated using the Voigt model. Odd numbers correspond to films ending in $\mathrm{CHI}$ and even numbers to films ending in ELR-RGD. Linear fitting matches well with the cumulative thickness variation $\left(r^{2}=0.99824\right)$. 
an approximate value of $40 \mathrm{~Hz}$. On the other hand, the $\Delta D$ increased, revealing that the film is not rigid and exhibits the typical viscoelastic behavior, a common situation when using macromolecular systems. ${ }^{[19 b, 20]}$

At the working $\mathrm{pH}$ of 5.5, we relied on the protonation state of the ELR-RGD aspartic acids: under such conditions, these residues are deprotonated and negatively charged $\left(\mathrm{pK}_{\mathrm{a}}\right.$ ranging from 3.0 to 4.7$).{ }^{[21]}$ This would allow them to interact by electrostatic self-assembly (ESA) with the positively charged amine groups of CHI. Prior to the monitoring, the $\zeta$-potential of $\mathrm{CHI}$ and ELR-RGD solutions was measured at working conditions $(\mathrm{pH}=5.5$ and ionic strength at $0.15 \mathrm{M})$, showing a charge of $+17.0 \pm 0.6 \mathrm{mV}$ and $-8.4 \pm 0.33 \mathrm{mV}$, respectively. ELRs are essentially hydrophobic by nature but can be modified to include specific charged residues. $\mathrm{CHI}$ is also insoluble in water and can only be dissolved at acidic conditions, below its $\mathrm{pK}_{\mathrm{a}}$ (approximately 6). ${ }^{[22]}$ It has been reported that electrostatic deposition demands appropriate matching of the charge density of the used polyelectrolyte pair, rather than a minimum charge density, ${ }^{[8 a, 23]}$ but in this case the $\zeta$-potential showed no charge balance (i.e., equal absolute values). Although ESA was a driving force of construction, other forces, such as hydrophobic interactions, are likely to participate in the assembling phenomenon. In fact, Figure 2a shows the successful combination of the used pair, at the assembly $\mathrm{pH}$ of 5.5 , near the $\mathrm{pK}_{\mathrm{a}}$ of CHI. We can assume that a fraction of already deprotonated amine groups interacts with the hydrophobic residues of the ELR-RGD structure and allow construction of the coating.

The QCM-D data was used to estimate the thickness variations of the film with each adsorption cycle. Figure $2 b$ shows the results calculated using the Voigt model, implemented in the Q-Tools software from Q-Sense. ${ }^{[24]}$ It can be observed that the thickness increased linearly during the buildup, in accordance with the evolution of $\Delta F_{v}$ and $\Delta D$ variations. After the deposition of 20 layers, the film had a thickness of $81 \mathrm{~nm}$, corresponding to an approximate total area density of $9.6 \mu \mathrm{g} \mathrm{cm}^{-2}$ (multiplication of thickness by the layer density, $1200 \mathrm{~kg} \mathrm{~m}^{-3}$, allowed for the best fitting). Figure $2 \mathrm{~b}$ also shows the thickness of each layer ("thickness increase" in the graphic). Overall, the increase of thickness is higher for the adsorption steps of ELR-RGD. This is the result of the lower charge exhibited by the recombinamer, which requires more material to overcompensate the charges of the underlying CHI ones. The results obtained through QCM-D measurements demonstrate that ELR-RGD can be used successfully with $\mathrm{CHI}$ to conceive a viscoelastic polymeric coating using the LbL approach.

\subsection{Characterization of the Self-Assembled Coatings}

When a material adheres to a substrate, it is expected that such phenomenon will result in physicochemical changes at the surface. ${ }^{[25]}$ The measurement of contact angles is an easy method that allows a quick assessment of the modification of a surface in terms of wettability. Its usefulness is even higher when such wettability is significantly different for each layer. Figure 3 shows the contact angle variation of the CHI/

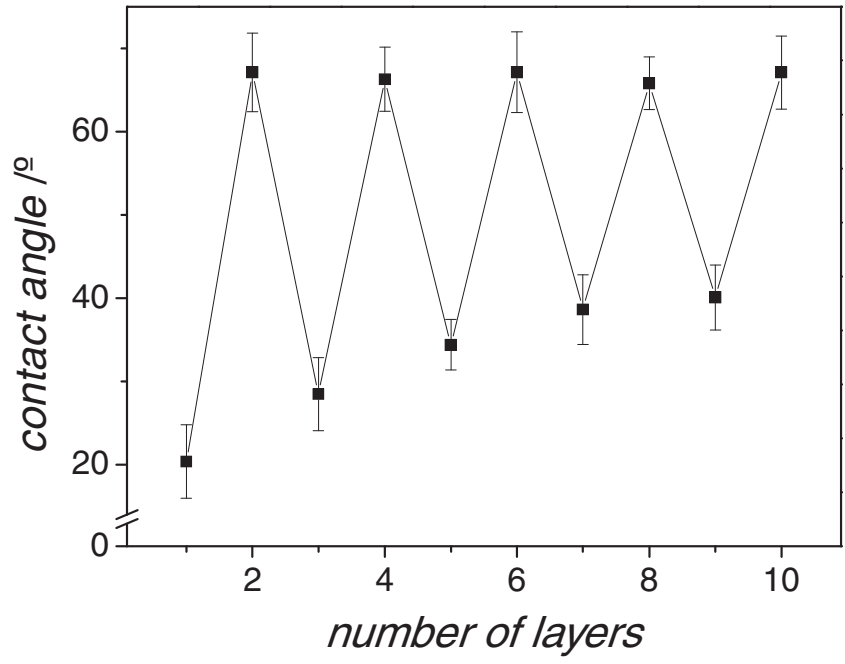

Figure 3. Evolution of the contact angle of the films obtained with sequential adsorption of $\mathrm{CHI}$ and ELR-RGD, up to 10 layers. Odd numbers correspond to films ending in $\mathrm{CHI}$ and even numbers to films ending in ELR-RGD. Error bars represent two standard deviations (number of layers, $n$, with $n=9$ ).

ELR-RGD film with the buildup, up to 10 layers. The films were adsorbed onto glass (contact angle of $(28.6 \pm 2.9)^{\circ}$ ). Each measurement was performed after $1 \mathrm{~h}$ of immersion in phosphate buffer saline (PBS) at $37{ }^{\circ} \mathrm{C}$. While films ending in ELR-RGD retained their moderate hydrophobic behavior (approximately between $62^{\circ}$ and $71^{\circ}$ ), the ones ending in $\mathrm{CHI}$ increased gradually, from $(20.4 \pm 4.4)^{\circ}$ in the first layer to $(40.1 \pm 3.9)^{\circ}$ in the fifth (layer 9).

The increasing values of contact angles obtained for CHIending films can be explained by the influence of the underlying adsorbed layers on the coating interface. When using weak polyelectrolytes, the most charged polymer tends to be adsorbed as a thinner layer with a flat chain conformation, while the less charged polymer tends to adsorb as a thicker layer, forming loopier-type structures. ${ }^{[26]}$ Since ELR-RGD is a modified version of the basic repeating block of elastin with charged amino acids, CHI appears as the most charged polymer, as already presented by the $\zeta$-potential measurements. Furthermore, the thickness increase of each layer displayed in Figure $2 b$ helps to demonstrate that the ELR-RGD layers are overall thicker than the ones of CHI. Thus, the surface properties of the $\mathrm{CHI}$ layer may reflect the effect of the underlying ELR one, even in terms of wettability, while the opposite does not occur.

The featured films were conceived using a recombinant ELR, a biomaterial exhibiting stimuli-responsive properties in solution. It would be interesting to observe how the wettability of the films behaves when they are exposed to several stimuli. To achieve this goal, we measured the contact angles at different values of temperature, $\mathrm{pH}$, and ionic strength (using $\mathrm{NaCl}$ as the ionic component) of 5 pairs of layers ending in ELR-RGD, labeled (CHI/ELR-RGD) $)_{5}$, assembled in glass substrates. After the assembly, the coatings were placed at different conditions of each studied stimulus (taking $25{ }^{\circ} \mathrm{C}$, $\mathrm{pH} 7.4$, and an ionic strength of $0.15 \mathrm{~m}$ as starting values), in order to determine if their variation triggered a transition 


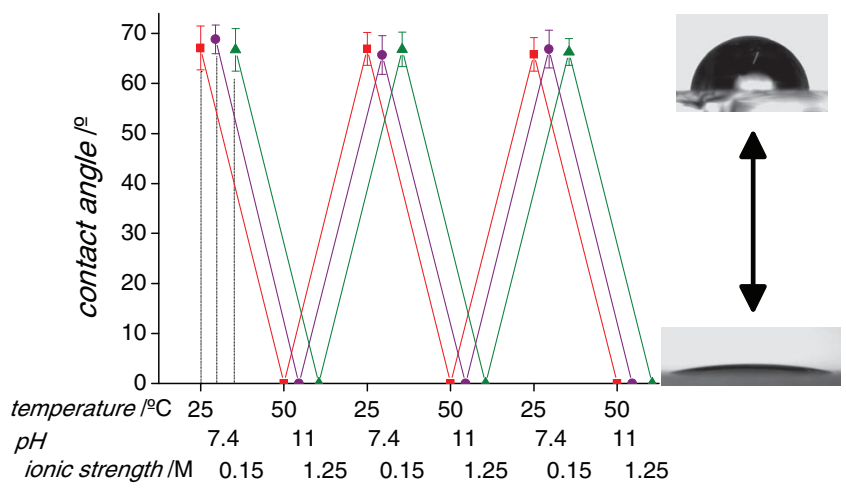

Figure 4. Contact angle measurements as a function of temperature (red), $\mathrm{pH}$ (purple), and ionic strength (green) in a (CHI/ELR-RGD) film. Three cycles are represented. Error bars represent two standard deviations $(n=9)$.

that could be detected by differences in wettability. Figure 4 shows the contact angle values measured upon cyclic variations of temperature $\left(25\right.$ to $\left.50{ }^{\circ} \mathrm{C}\right), \mathrm{pH}$ (7.4 to 11) and ionic strength $(0.15$ to $1.25 \mathrm{M})$.

Taking the case of the temperature at $25^{\circ} \mathrm{C}$, an initial wettability exhibiting values of $67.1 \pm 4.7$ was measured, corresponding to a moderate hydrophobic surface. However, upon increasing the temperature to $50{ }^{\circ} \mathrm{C}$, the contact angle values decreased to those of a superhydrophilic surface, corresponding to a totally wettable surface. ELRs show a phase transition in solution above $T_{\mathrm{t}}$ similar to the transition of other temperature-sensitive polymers with lower critical solution temperature (LCST). However, the hydrophobicity of such a surface did not increase with the switch to high temperatures. In fact, while the hydrophobicity of surfaces modified with LCST polymers increases, ${ }^{[4 b]}$ the behavior of ELRs when adsorbed onto a surface is opposite to the expected. This result is in accordance to the case of polymers with an upper critical solution temperature (UCST), which become more hydrophilic. ${ }^{27]}$ Such atypical wettability behavior of ELR-RGD temperature-responsive coatings has been previously observed in a thin ELR monolayer adsorbed onto CHI substrates. ${ }^{[16 b]}$ We have shown that upon the transition, the collapsed biopolymer aggregates into micelle-like structures, as suggested by topography measurements. They assume a conformation where the hydrophobic chains are directed to the inside and the charged residues to the outside of such organizations. Additionally, while the properties of such surfaces change at $50{ }^{\circ} \mathrm{C}$, the polymer in solution shows a transition at $23{ }^{\circ} \mathrm{C}$, where the association is much more facilitated than at the surface, to which the polymer is tightly connected.

Similar results to that of temperature were obtained for $\mathrm{pH}$ and ionic strength, even exhibiting similar contact angle values. In the case of $\mathrm{pH}$, ELR-RGD has lysines in its structure, which amine groups are protonated below the $\mathrm{pK}_{\mathrm{a}}$ values ranging from 9.4 to $10.6 .{ }^{[21]}$ Above the $\mathrm{pK}_{\mathrm{a}}$, the amine groups are deprotonated and the hydrophobicity of ELRRGD increases. It is known that the more hydrophobic the ELR, the lower the $T_{\mathrm{t}}$ in solution is. ${ }^{[28]}$ Thus, with the increase of $\mathrm{pH}$ the mean hydrophobicity of ELR-RGD also increases, up to a $\mathrm{pH}$ of 11 . At this value, the transition to superhydrophilicity was detected, as depicted in Figure 4. In the case of ionic strength, Reguera et al. suggested that an increase in the salt concentration increases the polarity of the solvent. This creates a higher difference in polarity with respect to the hydrophobic moieties of the polymer, causing more and more ordered structures surrounding the polymer chains. ${ }^{[29]}$ As observed, a transition was detected upon the increase of ionic strength to $1.25 \mathrm{M}$, which is in accordance with the behavior of this family of polymers. These results show that the wettability of $(\mathrm{CHI} / \mathrm{ELR}-\mathrm{RGD})_{5}$ films is strongly dependent not only on temperature, but also on $\mathrm{pH}$ and ionic strength, which influence the $T_{\mathrm{t}}$ of ELRs in aqueous solutions as well. Furthermore, the films were submitted to three cycles of variations and still retained full reversibility and similar contact angle values, showing that the films are robust and capable of withstanding sharp changes of the environment, while retaining functionality.

The results presented so far reflect the nature of the studied ELR, and characterize the conceived film as a stimuli-sensitive coating that could prove to be useful in the development of controlled release systems and in surfaces capable of controlling cell behavior. In order to fully understand this behavior, the wettability was further studied for several arrays of conditions, consisting of different combinations of $\mathrm{pH}$ and ionic strength at three relevant temperatures: $25^{\circ} \mathrm{C}$ (room temperature), $37^{\circ} \mathrm{C}$ (temperature of the body), and $50{ }^{\circ} \mathrm{C}$ (determined to be the temperature of transition of ELR-RGD at the surface). The diagram of Figure $\mathbf{5}$ is the graphical representation of the measurements performed in $(\mathrm{CHI} / \mathrm{ELR}-\mathrm{RGD})_{5}$, in an attempt to characterize the coating wettability and to define a processing window for these films.

To our knowledge, this is the first time that ELRadsorbed surfaces are extensively characterized for their responsive character towards external stimuli. There are

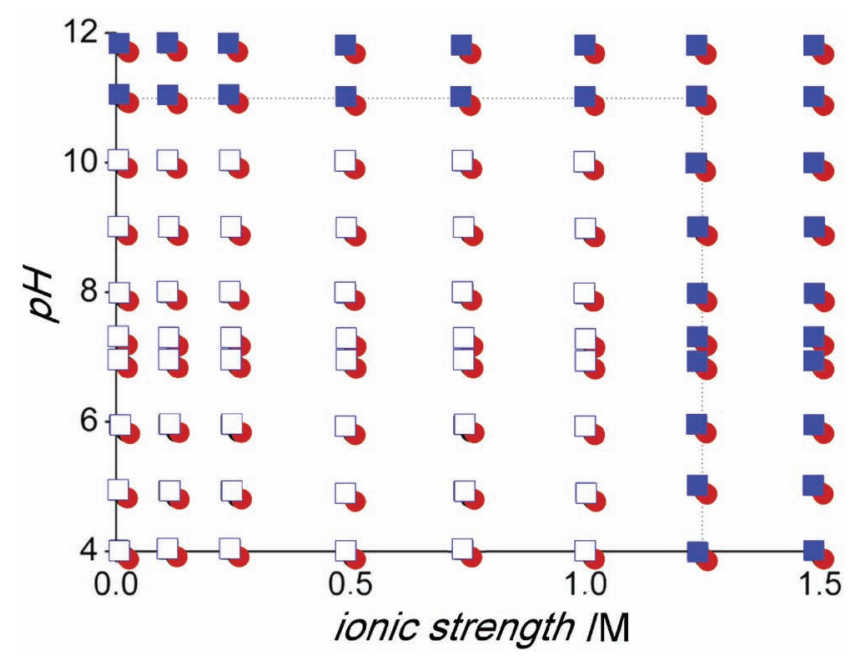

Figure 5. Map of the wettability of (CHI/ELR-RGD) 5 films as a function of condition arrays of temperature, $\mathrm{pH}$, and ionic strength stimuli. Open symbols stand for a moderate hydrophobic surface (contact angle of about $67^{\circ}$ ) and closed ones for a superhydrophilic surface. The blue squares represent measurements performed at 25 and $37^{\circ} \mathrm{C}$ and the red circles measurements at $50^{\circ} \mathrm{C}$. 
two areas or phases represented: i) one represented by open squares and full circles, and ii) the other represented by full squares and full circles. Blue squares stand for $25^{\circ} \mathrm{C} / 37^{\circ} \mathrm{C}$, while red circles stand for $50{ }^{\circ} \mathrm{C}$. Open and full symbols stand for a moderate hydrophobic (characteristic CA of $\left.(66.8 \pm 3.5)^{\circ}\right)$ or a completely wettable surface, respectively. The measurements performed have revealed the conditions of wettability transition for temperature, $\mathrm{pH}$, and ionic strength: $50{ }^{\circ} \mathrm{C}, 11$, and $1.25 \mathrm{M}$, respectively. The transition was also revealed to be rapid and independent of the contribution of the other stimuli: an "all-or-nothing" response towards external stimuli. In other words, the wettability of these systems shifts from moderate hydrophobicity to superhydrophilicity only when a stimulus equals or exceeds the abovementioned values, and there is no evidence of cross-dependencies between the three variables. Therefore, observing Figure 5, one can easily identify the parameters of transition at room temperature and $50{ }^{\circ} \mathrm{C}$. In the former, the wettability shifts at $\mathrm{pH} 11$ or ionic strength $1.25 \mathrm{M}$, as previously stated; in the latter, $50{ }^{\circ} \mathrm{C}$ is already the temperature at which the wettability shifts, so any combination of the other two stimuli can be disregarded.

The measurements performed at $37^{\circ} \mathrm{C}$ are of particular interest for biomedical applications, since ultimately these coatings are intended to be used in a biological environment. In the graphic, the wettability of the surfaces at $37{ }^{\circ} \mathrm{C}$ is similar to the case at room temperature, showing transitions dependent on $\mathrm{pH}$ and ionic strength at 11 and $1.25 \mathrm{~m}$, respectively. However, our measurements showed that in the range of $\mathrm{pH} 10-11$ and of ionic strength 1-1.25 $\mathrm{M}$, intermediate values could be observed (CAs of $\left.(30.5 \pm 20.6)^{\circ}\right)$. Such behavior is an interesting one: upon heating an ELR in solution above the $T_{\mathrm{t}}$, aggregation occurs and the solution becomes cloudy; given a few hours, or further increasing the temperature, the aggregates gradually settle and the result is a clean phase separation of solvent and polymer. ${ }^{[30]}$ At the studied surfaces, the equivalent to this "cloudy point" could only be observed for this small range of values and the wettability shift is only fully achieved-and quickly, not gradually as in solution-for $\mathrm{pH} 11$ and ionic strength $1.25 \mathrm{M}$, as is the case at room temperature. Thus, the diagram summarizes the response of the films ending in ELR-RGD as a function of three distinct variables, showing an independent and immediate effect of three of the parameters influencing the conformation of ELRs.

The molecular organization of a surface may give valuable information about the physical properties of a substrate. Figure 6 shows the surface roughness for the fifth pair of layers assembled in glass, ending in either CHI (layer 9) or ELR-RGD (layer 10), and AFM images of (CHI/ELRRGD) 5 films at physiological and transition $\mathrm{pH}$ values. An approach similar to that of contact angle measurements was followed: the samples were placed at room temperature in
PBS buffered at two distinct pHs, 7.4 and 11, and withdrawn for immediate topography image acquisition.

We expected that with increasing $\mathrm{pH}$ the surface roughness would increase as well, due to the triggering mechanism of CHI and ELR-RGD transitions above each $\mathrm{pK}_{\mathrm{a}}$. The AFM images in Figure 6 show the topography changes of (CHI/ ELR-RGD) 5 and reveal the formation of large and compact structures upon the aggregation of ELRs at the surface"micelle-like" structures which increases the water affinity at the interface by the exposure of hydrophilic groups. In fact, such organization is predicted even by the earliest theories of the natural elastin conformation. ${ }^{[31]}$

The analysis of the roughness showed that it increased with $\mathrm{pH}$, from $3.6 \pm 0.3$ to $4.9 \pm 0.3 \mathrm{~nm}$ (root-mean-square (RMS) roughness), showing that the topography of the films responded to $\mathrm{pH}$ changes, regardless of the last adsorbed layer. As a side note, the corresponding contact angle of the $(\mathrm{CHI} / \mathrm{ELR}-\mathrm{RGD})_{4} \mathrm{CHI}$ films displayed a value of $(64.3 \pm$ 3.6 $)^{\circ}$ upon increasing the $\mathrm{pH}$, higher than the value obtained for layer 9 due to the deprotonation of $\mathrm{CHI}$. Such a result reinforces the fact that the properties of a modified surface reflect mainly those of the material present at the interface, as is often expected after such an approach. The effect of $\mathrm{pH}$ on roughness was greater when the last layer was composed of ELR-RGD, as evidenced by the higher values displayed at each condition. Such structural changes were more visible when the ELR-RGD was deposited as the last layer, since the macromolecular segments exhibit more degrees of freedom for conformational rearrangements. $\mathrm{pH}$ and ionic strength may influence the electrostatic interactions between each layer, but similar results were obtained for temperature changes. We suggest that such similar results evidence that the aggregation of the ELR is the factor that contributes the most to the increase of roughness. Similar results were also obtained in previous studies. ${ }^{[16 b]}$

The rough character of the surface also contributes to the atypical extreme hydrophilic nature of the substrate, based on a simple lotus effect. In fact, the Wenzel model predicts 
that the change from a hydrophobic surface towards a more hydrophilic one is amplified in substrates exhibiting nano-/ microscale roughness. ${ }^{[32]}$

\subsection{Cell Culture Studies of SaOS-2 Osteoblast-Like Cells}

Smart surfaces have been used in different fields in situations where it is relevant to control the adhesion of cells or micro-organisms, protein adsorption or transport properties for the release of bioactive agents. In many cases, it is desirable that the substrate interacts favorably with cells. It has been reported that the RGD sequence is the minimal sequence required for recognition by cell membrane integrins, such as $\alpha 5 \beta 1$ and $\alpha \mathrm{V} \beta 3$ integrins. ${ }^{[33]}$ The affinity of cells for materials should be improved with the incorporation of a peptide having this sequence.

So far, we have been able to expand the concept of LbL to a class of materials that is not commonly used in literature. We also presented results showing that the films display distinct properties for different conditions of temperature, $\mathrm{pH}$, and ionic strength. This feature could be exploited to modulate protein and cell adhesion by playing with one of the mentioned stimuli. Unfortunately, the presented transition values are simply not suitable for most biological applications. We expect to take advantage of the recombinant nature of ELRs in the near future to produce films that could be triggered at lower values. Nonetheless, the biological performance of the current films was analyzed in order to determine if this kind of multilayer film is suitable for future tissue engineering devices with good response from cells, without any hazardous reaction.

In vitro studies were performed using SaOs-2 osteoblastlike cells. Although other possibilities could be explored, one of the uses we envisaged for these coatings is in orthopedic applications in order to improve host integration. To prove that the superior cell attachment was due to a specific interaction with RGD, the cell behavior was compared to surfaces with a negative control of ELR containing a scrambled RDG sequence, labeled $\mathrm{RDG}^{(-)}$, in the form of 5 bilayers with $\mathrm{CHI},\left(\mathrm{CHI} / \mathrm{ELR}-\mathrm{RDG}^{(-)}\right)_{5}$. The scrambled RDG sequence has been previously shown to have significantly less cellular adhesion ${ }^{[34]}$ and is similar to ELR-RGD in all aspects, except for the position of the G and D amino acids.

The SaOs-2 cell viability on ELR-RGD- and CHI-ending films- $(\mathrm{CHI} / \mathrm{ELR}-\mathrm{RGD})_{5}$ and $\quad(\mathrm{CHI} / \mathrm{ELR}-\mathrm{RGD})_{4} / \mathrm{CHI}$ respectively - was assessed at different time points. Independently of the wettability changes of the coatings, cell culture was performed at $37^{\circ} \mathrm{C}$, at which the surface is moderately hydrophobic (see Figure 5), under serum-free conditions. Cell viability and activity was assessed through (3-(4,5-dimethylthiazol-2-yl)-5-(3-carboxymethoxyphenyl)-2-(4-sulfophenyl)2H-tetrazolium) (MTS) and alkaline phosphatase (ALP) assays. A qualitative evaluation of the cell number attached to the surfaces was performed using fluorescence microscopy and with 4',6-diamidino-2-phenylindol (DAPI) and phalloidin staining of cells.
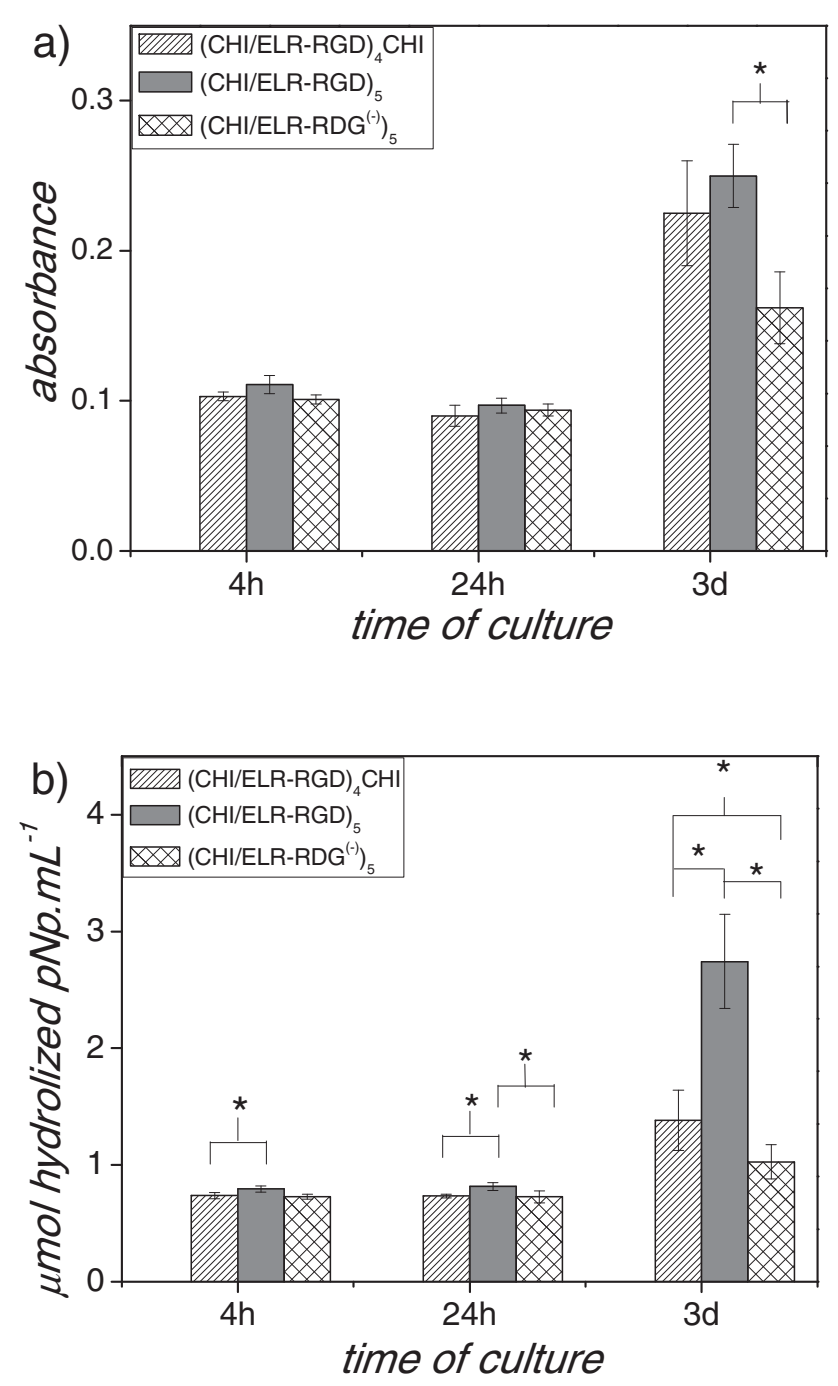

Figure 7. In vitro tests on films ending in CHI, ELR-RGD, and ELR-RDG ${ }^{(-)}$. a) Cell viability of SaOs- 2 cells for 4 h, 24 h, and 3 days. b) ALP assays on the studied films for $24 \mathrm{~h}$ and 3 days. Data are means \pm SD $(n=3$; $* p<0.05)$.

Figure 7a shows the MTS assay in the different surfaces after 4 h, 24 h, and 3 days (note: contact angles showed that the films still retain their properties during the studied timeframe). The results show that, for every time point, all tested samples were noncytotoxic towards the SaOs- 2 cell line, and therefore the materials used to construct the multilayers did not cause any toxicity problems to the attached cells nor to the cells suspended in culture. Among the various biological functions of osteoblasts, secretion of ALP is an important indicator determining the activity of the cells. The statistical analysis of the data (Figure 2b) shows that ALP activity was stimulated by $(\mathrm{CHI} / \mathrm{ELR}-\mathrm{RGD})^{5}$ coatings after $24 \mathrm{~h}$ in culture. After 3 days, the differences in cell activity became more evident in the surface with ELR-RGD in comparison to films ending in ELR-RDG ${ }^{(-)}$or CHI.

Differences in the number of cells attached to the different surfaces were also observed, as seen in Figure 8. Comparing the microscopy images, after $4 \mathrm{~h}$ of incubation, ELRRGD surfaces show a significant attached number of cells, 
$(\mathrm{CHI} / \mathrm{ELR}-\mathrm{RGD})_{5} \quad(\mathrm{CHI} / \mathrm{ELR}-\mathrm{RGD})_{4} \mathrm{CHI} \quad\left(\mathrm{CHI} / \mathrm{ELR}-\mathrm{RDG}{ }^{(-)}\right)_{5}$
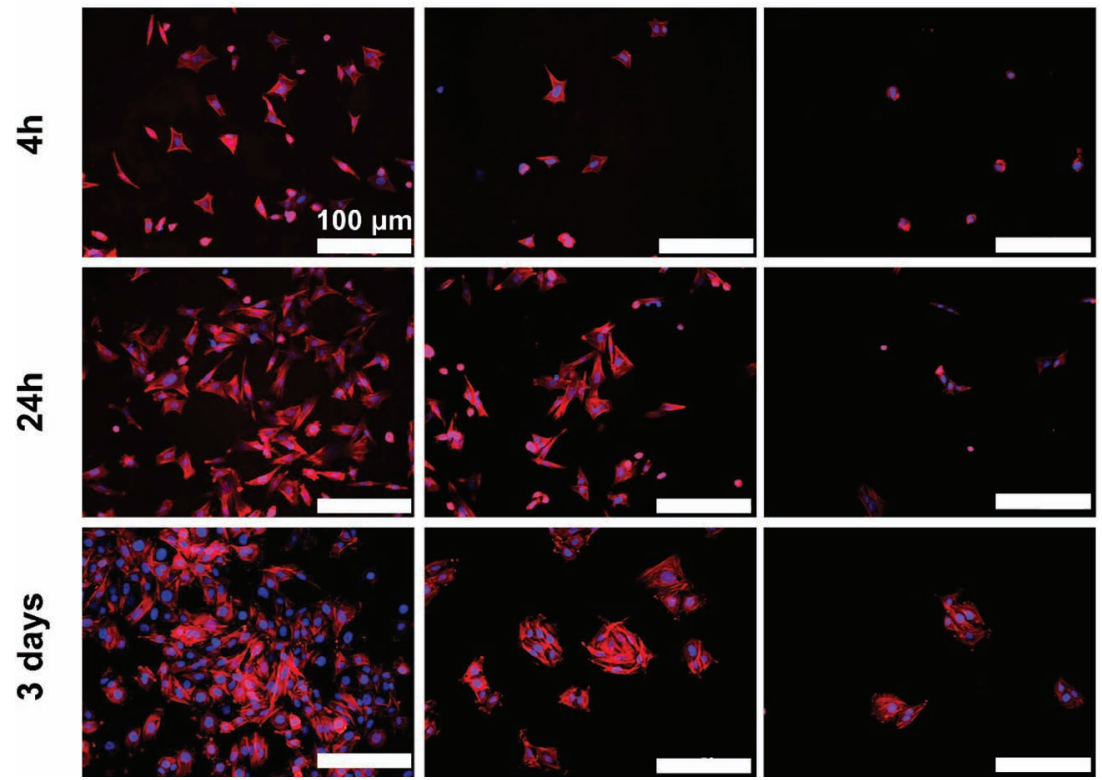

Figure 8. DAPI (blue) and phalloidin (red) staining of the studied cells cultured for distinct times on the studied multilayer substrates, ending in CHI, ELR-RGD, and ELR-RDG( ${ }^{-)}$. Tests performed at $4 \mathrm{~h}, 24 \mathrm{~h}$, and 3 days. Scale bar: $100 \mu \mathrm{m}$.

compared with nonmodified surfaces or the negative analog RDG. Based on the cell densities of the samples at $24 \mathrm{~h}$ and 3 days, it is possible to verify that (CHI/ELR-RGD) $)_{5}$ coatings demonstrated a very significant cell proliferation in comparison to the coatings ending in $\mathrm{CHI}$ and in $(\mathrm{RDG})^{(-)}$. Besides the higher number of cells on the surfaces with RGD, cells seeded on those substrates spread significantly more than the ones on chitosan or the negative control. Thicker and wellorganized filamentous actin bundles were observed in cells growing on RGD surfaces.

From our results, adsorption of ELR-RGD has shown to enhance cell adhesion and activity when compared to the CHI-ending films substrates and the scrambled RDG sequence, showing good in vitro performance of the presented films.

\section{Conclusion}

Our results show that ELRs containing biologically relevant peptide sequences can be successfully combined with $\mathrm{CHI}$ in a sequential multilayer approach based on electrostatic and hydrophobic interactions. QCM-D results allowed us to study the construction of the polymeric films and its properties, showing the buildup of a film with a linear growth. Although glass was used to assemble such coatings, it is expected in the future that such straightforward surface modification techniques will allow us to coat other substrates with more complex geometries, including scaffolds or particles. The stimuli-responsive properties of the films were extensively studied, showing acute and independent cyclic response towards temperature, $\mathrm{pH}$, and ionic strength; the values of transition for each stimulus were identified: $50{ }^{\circ} \mathrm{C}$,
11, and $1.25 \mathrm{M}$, respectively. Below each critical transition point, the surfaces were more hydrophobic. Above those, the surface acquired a very hydrophilic character due to the biopolymer chain collapse and subsequent exposure of charged residues to the outer region of the coating. The transition was also evident in the changes in the topography of the surfaces as we increased the $\mathrm{pH}$ of the film above the transition values, increasing their roughness due to the aggregation of ELR chains on the top layer.

SaOs-2 osteoblast-like cells were used to test the biological performance of the multilayered system studied in vitro of the films. The cells showed increased adhesion and activity with ELR-RGD ending coatings, in comparison to the ones ending with both $\mathrm{CHI}$ and the nonbioactive ELR-RDG( ${ }^{(-)}$. This work demonstrates the potential to use natural and nature-inspired macromolecules to modify surfaces taking advantage of a simple layer-by-layer technique, which can be further extrapolated to more complex devices. The potential of this technology can further stimulate the development of other similar biomedical products in the near future, such as devices entirely processed through layerby-layer for the delivery of pharmaceuticals, cell culture, or structures for tissue engineering.

\section{Experimental Section}

ELR-RGD Expression and Purification: Expression conditions and purification protocols for ELR-RGD were adapted from Meyer and Chilkoti ${ }^{[35]}$ and Girotti et al. ${ }^{[28]}$ Gene expression of a recombinant Escherichia coli strain BLR (DE3) containing the expressing gene of ELR-RGD was induced in a $12 \mathrm{~L}$ Applikon fermenter, in terrific broth medium (TB) with carbenicilin $(0.1 \% \mathrm{v} / \mathrm{v})$ and glucose $(0.1 \% \mathrm{v} / \mathrm{v})$, under controlled conditions of temperature $\left(37^{\circ} \mathrm{C}\right)$ and $\mathrm{pH}$ (7.00). The fermentation was stopped after registering an optical density variation at $600 \mathrm{~nm}$ inferior to 0.25 , in a time frame of $1 \mathrm{~h}$. Subsequent to fermentation, the culture was harvested by centrifugation, resuspended, and lysed by ultrasonic disruption. Insoluble debris was removed by centrifugation and the cleared lysate was subjected to several cycles of cold and warm centrifugations, of 4 and $40{ }^{\circ} \mathrm{C}$, respectively. All the purification steps were carried out in a sodium chloride solution $(0.5 \mathrm{~m})$. The polymer in solution was then frozen at $-24^{\circ} \mathrm{C}$ and freeze-dried.

ELR-RGD Characterization: Differential Scanning Calorimetry (DSC) experiments were performed on a Mettler Toledo $822 \mathrm{e}$ (United States) with a liquid nitrogen cooler accessory. Both temperature and enthalpy were calibrated with an indium standard at the same experimental conditions used for the studied materials. Water solutions of H-RGD6 (50 $\mathrm{mg} \mathrm{mL}^{-1}$ ) were prepared at different values of $\mathrm{pH}$. In a typical DSC run, a small volume of solution $(20 \mu \mathrm{L})$ was placed inside a standard aluminum pan hermetically 
sealed $(40 \mu \mathrm{L})$. The same volume of water was placed in the reference pan. All samples were equilibrated for $5 \mathrm{~min}$ at $5{ }^{\circ} \mathrm{C}$ inside the sample chamber just before the beginning of each experiment. A heating rate of $5^{\circ} \mathrm{C} \mathrm{min}^{-1}$ was used. Transition temperatures and enthalpy values were measured as a function of $\mathrm{pH}$. To assess the biopolymer molecular weight, MALDI-TOF mass spectroscopy was performed in a Voyager STR, from Applied Biosystems, in linear mode and with an external calibration using bovine serum albumin (BSA). Amino acid composition determination was previously used to assess the composition of ELRs. ${ }^{[14]}$

Buildup and Characterization of the Self-Assembled Coatings: Medium-molecular-weight grade $\mathrm{CHI}$ was purchased from Sigma and purified. Solutions of purified CHI and ELR-RGD $\left(0.1 \mathrm{mg} \mathrm{mL}^{-1}\right)$ containing $\mathrm{NaCl}(0.15 \mathrm{M})$ were prepared. The $\mathrm{pH}$ of the solutions was adjusted to 5.5 . The $\zeta$-potential of each solution was determined using a Nano-ZS from Malvern (United Kingdom), at $25^{\circ} \mathrm{C}$. A Q-Sense E4 quartz-crystal microbalance (Q-Sense AB, Sweden) with dissipation monitoring system was used for monitoring in situ the deposition of CHI/ELR-RGD multilayers at the surface of goldcoated crystals. This technique has previously been extensively described. ${ }^{[19]}$ Very briefly, AT cut quartz crystal can be excited at its fundamental frequency $(5 \mathrm{MHz})$ and at several overtones: 25 , 35 , and $45 \mathrm{MHz}$ (fifth, seventh, and ninth overtones, respectively). When a thin film is deposited onto the sensor crystal the frequency decreases. If the film is thin and rigid the decrease in frequency $(\Delta F)$ is proportional to the mass of the film. However, when using polymers, the adsorbed film is not rigid and this relation is not valid: there is a loss of energy stored in each vibrational cycle and the film exhibits the typical viscoelastic behavior, which is evident by the change in the dissipation $(\Delta D)$. The crystals were first cleaned in an ultrasound bath at $30{ }^{\circ} \mathrm{C}$, and immersed successively in acetone, ethanol, and isopropanol. Adsorption took place at $25{ }^{\circ} \mathrm{C}$ and $\mathrm{pH} 5.5$ and at a constant flow rate of $50 \mathrm{~mL} \mathrm{~min}{ }^{-1}$. Starting with the $\mathrm{CHI}$ solution, each one was pumped into the system for $10 \mathrm{~min}$. Each deposition cycle was followed by a rinsing step $(\mathrm{NaCl}, 0.15 \mathrm{M})$ at $\mathrm{pH} 5.5$, for $10 \mathrm{~min}$, and $\Delta F_{v}$ and $\Delta D$ were monitored in real time. The frequency of each overtone was normalized to the fundamental resonant frequency of the quartz crystal, by dividing it by $v$ (where $v=5 ; 7 ; 9$ ). The thickness of the films was estimated using the Voigt model through the Q-Tools software, from Q-Sense. ${ }^{[2]}$ The area density was calculated by multiplying the thickness by the layer density $\left(1200 \mathrm{~kg} \mathrm{~m}^{-3}\right.$ led to the best fit).

Contact-Angle Measurements: PBS contact angles were measured in a DataPhysics OCA15 system. The coatings were prepared at the surface of regular glass microscopy slides, from Marienfeld (Germany). The glass was cleaned using the same protocol to which QCM-D crystals were submitted. Coatings up to 10 layers -5 pairs of $\mathrm{CHI}$ and ELR-RGD-were characterized for their wettability for each layer assembled, at $37^{\circ} \mathrm{C}$ and $\mathrm{pH}$ 7.4. An approach described by Park, Draper, and Flynn was used to measure contact angles dependent on several temperature, $\mathrm{pH}$, and ionic strength conditions. ${ }^{[36]}$ The measurements were performed for a $\mathrm{pH}$ range from 4 to 12 , ionic strength from 0 to $1.5 \mathrm{~m}$ of $\mathrm{NaCl}$, and three relevant temperatures: 25,37 , and $50^{\circ} \mathrm{C}$. The samples were immersed in a beaker of PBS placed under each condition. After $1 \mathrm{~h}$, the samples were withdrawn and contact angles were measured. The time of $1 \mathrm{~h}$ was determined to be enough to have a stable surface. Upon determining the wettability transition parameters, three cycles were performed by changing the transition values above and below each transition value.

Atomic Force Microscopy Measurements: AFM measurements were performed in a MultiMode STM microscope controlled by the NanoScope III from Digital Instruments system, operating in tapping mode at a frequency of $1 \mathrm{~Hz}$. Glass slides were cut into pieces of $1 \mathrm{~cm} \times 1 \mathrm{~cm}$ and used as substrates for multilayer adsorption. Surfaces coated with 5 pairs of $\mathrm{CHI} /$ ELR-RGD were immersed in a PBS for $30 \mathrm{~min}$. To assess the influence of $\mathrm{pH}$ in the coatings, a similar protocol to the contact angle measurements was followed. The samples were immersed in PBS at two pH values: 7.4 and 11. To avoid the formation of crystals during the acquisition, the samples were immersed shortly in ultrapure water for removing excess of saline solution. The samples were then retrieved and introduced in the device. The analyzed area was $1 \mu \mathrm{m} \times 1 \mu \mathrm{m}$.

Cell Culture Studies of SaOS-2 Osteoblast-Like Cells: Cell studies were performed onto coatings of 5 pairs of layers, ending in ELR-RGD, CHI, or ELR-RDG(-) (negative control), assembled at the surface of glass coverslips with $1 \mathrm{~cm}$ of diameter. Tissue culture polystyrene (TCPS) was used as a positive control. Cell adhesion was studied using the SaOs-2 osteoblast-like osteosarcoma cell line. Cells were seeded on the surfaces $\left(5 \times 10^{4}\right.$ cell $\left.\mathrm{mL}^{-1}\right)$ and incubated in serum-free Dulbecco's Modified Eagle's medium (DMEM), loaded antibiotics/antimicotics (1\%) at $37{ }^{\circ} \mathrm{C}, 5 \% \mathrm{CO}_{2}$. The substrates were analyzed at different time points: $4 \mathrm{~h}, 24 \mathrm{~h}$, and 3 days. At each time point, cells on the coated surfaces were washed with PBS twice, they were fixed in formalin (3.7\%), the nuclei of the cells stained with DAPI, and the actin was stained with phalloidin.

For ALP assays, samples collected on each time point, were washed with PBS and immersed in $1 \mathrm{~mL}$ of ultrapure water to lyse the cells. To each well of a 96-well plate were added the sample $(20 \mu \mathrm{L})$ plus substrate solution $(60 \mu \mathrm{L}, 0.2 \%(\mathrm{w} / \mathrm{v}) p$-nitrophenyl (pNP) phosphate/ $1 \mathrm{~m}$ diethanolamine $\mathrm{HCl}$, at $\mathrm{pH}$ 9.8) The plate was then incubated in the dark for $45 \mathrm{~min}$ at $37^{\circ} \mathrm{C}$. After the incubation period, a stop solution ( $80 \mu \mathrm{L}, 2 \mathrm{~m} \mathrm{NaOH} / 0.2 \mathrm{~mm}$ EDTA (ethylenediaminetetraacetic acid)), was added to each well. The absorbance was then measured at $405 \mathrm{~nm}$ using $p$-nitrophenol as standard. For each experiment, a standard curve was constructed from reference suspensions at known cell concentrations. To access cell viability was performed MTS assay. MTS is bioreduced by cells. The values for absorbance of this reduced product at $490 \mathrm{~nm}$ are directly proportional to the number of living cells in the substrates.

\section{Acknowledgements}

We acknowledge the financial support through Fundação para a Ciência e Tecnologia (FCT) from Portugal (PhD grants SFRH/ $B D / 61126 / 2009$ and SFRH/BD/61390/2009), the European regional development fund (ERDF) from EU, from the MICINN (projects MAT 2007-66275-C02-01, MAT 2007-61604, MAT 200914195-C03-03, and PSE-300100-2006-1), the JCyL (projects VA034A09 and VA030A08), the CIBER-BBN (project CB06-010003), the JCYL and the Instituto de Salud Carlos III under the "Network Center of Regenerative Medicine and Cellular Therapy of Castilla and León". 
[1] a) D. Falconnet, G. Csucs, H. Michelle Grandin, M. Textor, Biomaterials 2006, 27, 3044-3063; b) A. L. Hook, N. H. Voelcker, H. Thissen, Acta Biomater. 2009, 5, 2350-2370; c) M. Tirrell, E. Kokkoli, M. Biesalski, Surf. Sci. 2002, 500, 61-83.

[2] a) N. M. Alves, I. Pashkuleva, R. L. Reis, J. F. Mano, Small 2010, 6, 2208-2220; b) S. Levenberg, R. Langer, Curr. Top. Dev. Biol. 2004, 61, 113-134; c) M. M. Stevens, J. H. George, Science 2005, 310, $1135-1138$

[3] J. Shi, N. Alves, J. Mano, Adv. Funct. Mater. 2007, 17, 3312-3318.

[4] a) R. M. da Silva, P. M. López-Pérez, C. Elvira, J. F. Mano, J. S. Román, R. L. Reis, Biotechnol. Bioeng. 2008, 101, 13211331; b) T. Okano, N. Yamada, H. Sakai, Y. Sakurai, Journal of Biomedical Materials Research 1993, 27, 1243-1251.

[5] F. Xia, H. Ge, Y. Hou, T. Sun, L. Chen, G. Zhang, L. Jiang, Adv. Mater. 2007, 19, 2520-2524.

[6] E. Delamarche, D. Juncker, H. Schmid, Adv. Mater. 2005, 17 , 2911-2933.

[7] H. G. Schild, Prog. Polym. Sci. 1992, 17, 163-249.

[8] a) P. Bertrand, A. Jonas, A. Laschewsky, R. Legras, Macromol. Rapid Commun. 2000, 21, 319-348; b) G. Decher, Science 1997, 277, 1232-1237; c) P. Sher, C. A. Custódio, J. F. Mano, Small 2010, 6, 2644-2648.

[9] a) T. Boudou, T. Crouzier, K. Ren, G. Blin, C. Picart, Adv. Mater. 2010, 22, 441-467; b) T. Crouzier, K. Ren, C. Nicolas, C. Roy, C. Picart, Small 2009, 5, 598-608; c) A. Shukla, S. N. Avadhany, J. C. Fang, P. T. Hammond, Small 2010, 6, 2392-2404; d) Z. Tang, Y. Wang, P. Podsiadlo, N. Kotov, Adv. Mater. 2006, 18, 3203-3224.

[10] M. A. Meyers, P.-Y. Chen, A. Y.-M. Lin, Y. Seki, Prog. Mater Sci. 2008, 53, 1-206

[11] a) D. E. Meyer, A. Chilkoti, Biomacromolecules 2002, 3, 357367; b) J. C. Rodríguez-Cabello, L. Martín, M. Alonso, F. J. Arias, A. M. Testera, Polymer 2009, 50, 5159-5169; c) D. W. Urry, Angew. Chem. Int. Ed. 1993, 32, 819-841.

[12] J. C. Rodríguez-Cabello, M. Alonso, M. I. Díez, M. I. Caballero, M. M. Herguedas, Macromol. Chem. Phys. 1999, 200, 1831-1838.

[13] a) F. Arias, V. Reboto, S. Martín, I. López, J. Rodríguez-Cabello, Biotechnol. Lett. 2006, 28, 687-695; b) A. Nicol, D. Channe Gowda, D. W. Urry, J. Biomed. Mater. Res. 1992, 26, 393-413.

[14] A. Girotti, J. Reguera, J. Rodríguez-Cabello, F. Arias, M. Alonso, A. Testera, J. Mater. Sci. Mater. Med. 2004, 15, 479-484.

[15] M. Swierczewska, C. S. Hajicharalambous, A. V. Janorkar, Z. Megeed, M. L. Yarmush, P. Rajagopalan, Acta Biomater. 2008, 4, 827-837.

[16] a) J. Barbosa, R. Costa, A. Testera, M. Alonso, J. Rodríguez-Cabello, J. Mano, Nanoscale Res. Lett. 2009, 4, 1247-1253; b) R. R. Costa,
C. A. Custódio, A. M. Testera, F. J. Arias, J. C. Rodríguez-Cabello, N. M. Alves, J. F. Mano, Adv. Funct. Mater. 2009, 19, 3210-3218.

[17] a) N. M. Alves, J. F. Mano, Int. J. Biol. Macromol. 2008, 43, 401414; b) M. N. V. Ravi Kumar, React. Funct. Polym. 2000, 46, 1-27.

[18] a) N. Cini, T. Tulun, G. Decher, V. Ball, J. Am. Chem. Soc. 2010, 132, 8264-8265; b) R. V. Klitzing, Phys. Chem. Chem. Phys. 2006, 8, 5012-5033.

[19] a) F. Hook, B. Kasemo, T. Nylander, C. Fant, K. Sott, H. Elwing, Anal. Chem. 2001, 73, 5796-5804; b) K. A. Marx, Biomacromolecules 2003, 4, 1099-1120; c) S. M. Notley, M. Eriksson, L. Wågberg, J. Colloid Interface Sci. 2005, 292, 29-37.

[20] C. Picart, P. Lavalle, P. Hubert, F. J. G. Cuisinier, G. Decher, P. Schaaf, J. C. Voegel, Langmuir 2001, 17, 7414-7424.

[21] L. A. Fothergill-Gilmore, in Protein Biotechnology: Isolation, Characterization, and Stabilization, (Ed: F. Franks), Humana Press, Totowa NJ, USA 1993, 254-256.

[22] M. G. Peter, J. Macromol. Sci., Part A: Pure Appl. Chem. 1995, 32, 629-640.

[23] P. Fischer, A. Laschewsky, E. Wischerhoff, X. Arys, A. Jonas, R. Legras, Macromol. Symp. 1999, 137, 1-24.

[24] M. V. Voinova, M. Rodahl, M. Jonson, B. Kasemo, Phys. Scr. 1999, 59, 391.

[25] a) J. Mano, Adv. Eng. Mater. 2008, 10, 515-527; b) H. Noh, E. A. Vogler, Biomaterials 2006, 27, 5801-5812.

[26] a) J. Fu, J. Ji, W. Yuan, J. Shen, Biomaterials 2005, 26, 6684-6692; b) D. Yoo, S. S. Shiratori, M. F. Rubner, Macromolecules 1998, 31, 4309-4318.

[27] O. Azzaroni, A. A. Brown, W. T. S. Huck, Angew. Chem. Int. Ed. 2006, 118, 1802-1806.

[28] A. Girotti, J. Reguera, F. J. Arias, M. Alonso, A. M. Testera, J. C. Rodríguez-Cabello, Macromolecules 2004, 37, 3396-3400.

[29] J. Reguera, D. W. Urry, T. M. Parker, D. T. McPherson, J. C. Rodríguez-Cabello, Biomacromolecules 2007, 8, 354-358.

[30] D. W. Urry, J. Phys. Chem. B 1997, 101, 11007-11028.

[31] T. Weis-Fogh, S. Andersen, Nature 1970, 227, 718-721.

[32] R. N. Wenzel, Ind. Eng. Chem. 1936, 28, 988-994.

[33] a) M. C. Berg, S. Y. Yang, P. T. Hammond, M. F. Rubner, Langmuir 2004, 20, 1362-1368; b) P.-H. Chua, K.-G. Neoh, E.-T. Kang, W. Wang, Biomaterials 2008, 29, 1412-1421; c) E. Ruoslahti, Annu. Rev. Cell. Dev. Biol. 1996, 12, 697-715.

[34] J. C. Liu, D. A. Tirrell, Biomacromolecules 2008, 9, 2984-2988.

[35] D. E. Meyer, A. Chilkoti, Nat. Biotech. 1999, 17, 1112-1115.

[36] E. J. Park, D. D. Draper, N. T. Flynn, Langmuir 2007, 23, 7083-7089.

Received: May 5, 2011

Published online: August 2, 2011 